\title{
A class of groups for which every action is $\mathrm{W}^{*}$-superrigid
}

\author{
Cyril Houdayer ${ }^{1}$, Sorin Popa $^{2}$ and Stefaan Vaes ${ }^{3}$
}

\begin{abstract}
We prove the uniqueness of the group measure space Cartan subalgebra in crossed products $A \rtimes \Gamma$ covering certain cases where $\Gamma$ is an amalgamated free product over a nonamenable subgroup. In combination with Kida's work we deduce that if $\Sigma<\operatorname{SL}(3, \mathbb{Z})$ denotes the subgroup of matrices $g$ with $g_{31}=g_{32}=0$, then any free ergodic probability measure preserving action of $\Gamma=\operatorname{SL}(3, \mathbb{Z}) *_{\Sigma} \operatorname{SL}(3, \mathbb{Z})$ is stably $\mathrm{W}^{*}$-superrigid. In the second part we settle a technical issue about the unitary conjugacy of group measure space Cartan subalgebras.
\end{abstract}

Mathematics Subject Classification (2010). 46L36, 20E06, 37A20.

Keywords. $\mathrm{W}^{*}$-superrigidity, deformation/rigidity theory, $\mathrm{II}_{1}$ factor, ergodic equivalence relation, amalgamated free product group.

\section{Introduction}

This short article is a two-fold complement to [PV09]. The main result of [PV09] provides a class $\mathcal{E}$ of groups $\Gamma$ such that for every free ergodic probability measure preserving (pmp) action $\Gamma \curvearrowright(X, \mu)$, the $\mathrm{II}_{1}$ factor $\mathrm{L}^{\infty}(X) \rtimes \Gamma$ has a unique group measure space Cartan subalgebra up to unitary conjugacy. The class $\mathcal{E}$ contains all non-trivial amalgamated free products $\Gamma=\Gamma_{1} *{ }_{\Sigma} \Gamma_{2}$ such that $\Gamma$ admits a nonamenable subgroup with the relative property (T) and such that $\Sigma$ is an amenable group that is sufficiently non normal in $\Gamma$. In combination with known orbit equivalence superrigidity theorems, several group actions $\Gamma \curvearrowright(X, \mu)$ are shown in [PV09] to be $\mathrm{W}^{*}$-superrigid: any isomorphism between $\mathrm{L}^{\infty}(X) \rtimes \Gamma$ and an arbitrary group measure space $\mathrm{II}_{1}$ factor $\mathrm{L}^{\infty}(Y) \rtimes \Lambda$, comes from a conjugacy of the actions. For example the Bernoulli action $\Gamma \curvearrowright[0,1]^{\Gamma}$ is $\mathrm{W}^{*}$-superrigid for many of the groups $\Gamma \in \mathcal{E}$, see [PV09], Theorem 1.3. Using Kida's [Ki09], Theorem 1.4, and denoting by $\Sigma<$ $\mathrm{SL}(3, \mathbb{Z})$ the subgroup of upper triangular matrices, one deduces $\mathrm{W}^{*}$-superrigidity

\footnotetext{
${ }^{1}$ The first author was partially supported by ANR grant AGORA.

${ }^{2}$ The second author was partially supported by NSF Grant DMS-0601082.

${ }^{3}$ The third author was partially supported by ERC Starting Grant VNALG-200749, Research Programme G.0639.11 of the Research Foundation - Flanders (FWO) and KU Leuven BOF research grant OT/08/032.
} 
for every free ergodic pmp action $\Gamma \curvearrowright(X, \mu)$ such that all finite index subgroups of $\Sigma$ still act ergodically, see [PV09], Theorem 6.2.

In the first part of this article we generalize the uniqueness of the group measure space Cartan subalgebra to the case where $\Gamma=\Gamma_{1} *_{\Sigma} \Gamma_{2}$ is an amalgamated free product over a possibly non-amenable subgroup $\Sigma$, see Theorem 5 . We still assume some softness on $\Sigma$ by imposing the existence of a normal tower $\{e\}=\Sigma_{0} \triangleleft \Sigma_{1} \triangleleft$ $\cdots \triangleleft \Sigma_{n-1} \triangleleft \Sigma_{n}=\Sigma$ such that all quotients $\Sigma_{i} / \Sigma_{i-1}$ have the Haagerup property. We have to strengthen however the rigidity assumption by imposing that $\Gamma$ admits an infinite subgroup that has property (T). The proof of Theorem 5 is identical to the proof of [PV09], Theorem 5.2, apart from the fact that we need a new transfer of rigidity lemma, see Lemma 4 (cf. [PV09], Lemma 3.1).

Using Kida's [Ki09], Theorem 9.11, it follows that if $\Sigma<\mathrm{SL}(3, \mathbb{Z})$ denotes the subgroup of matrices $g$ with $g_{31}=g_{32}=0$, then any free ergodic pmp action of $\Gamma$ on $(X, \mu)$ is stably $\mathrm{W}^{*}$-superrigid, see Theorem 3. Contrary to the case where $\Sigma$ consists of the upper triangular matrices, no ergodicity assumption has to be made on the action of the finite index subgroups of $\Sigma$.

In the second part of this article we provide a detailed argument for the following principle: if $B \subset A \rtimes \Gamma$ is a Cartan subalgebra in a group measure space $\mathrm{II}_{1}$ factor and if $B$ embeds into $A \rtimes \Sigma$ for a sufficiently non normal subgroup $\Sigma<\Gamma$, then $B$ and $A$ are unitarily conjugate. So Proposition 8 provides a justification for the end of the proofs of [PV09], Theorems 5.2 and 1.4, which were rather brief compared to the rest of that article. We are very grateful to Steven Deprez who pointed out to us the necessity of adding more details.

\section{Preliminaries}

Intertwining-by-bimodules. Let $(M, \tau)$ be a von Neumann algebra with a faithful normal tracial state $\tau$ and let $A, B \subset M$ be (possibly non-unital) von Neumann subalgebras. In [Po03], Section 2, the technique of intertwining-by-bimodules was introduced. It is shown there that the following two conditions are equivalent.

- There exist projections $p \in A, q \in B$, a non-zero partial isometry $v \in p M q$ and a normal $*$-homomorphism $\theta: p A p \rightarrow q B q$ satisfying $x v=v \theta(x)$ for all $x \in p A p$.

- There is no sequence of unitaries $\left(w_{n}\right)$ in $A$ such that $\left\|E_{B}\left(a w_{n} b\right)\right\|_{2} \rightarrow 0$ for all $a, b \in M$.

If one, and hence both, of these conditions hold, we write $A \prec_{M} B$. By [Po01], Theorem A.1, if $A$ and $B$ are Cartan subalgebras of a $\mathrm{II}_{1}$ factor $M$, then $A \prec_{M} B$ if and only if $A$ and $B$ are unitarily conjugate.

Property (T) for von Neumann algebras. Let $(P, \tau)$ be a von Neumann algebra with a faithful normal tracial state $\tau$. A normal completely positive map $\varphi: P \rightarrow P$ is 
said to be subunital if $\varphi(1) \leq 1$ and subtracial if $\tau \circ \varphi \leq \tau$. Following [CJ85], we say that $P$ has property (T) if every sequence of normal subunital subtracial completely positive maps $\varphi_{n}: P \rightarrow P$ converging to the identity pointwise in $\|\cdot\|_{2}$, converges uniformly in $\|\cdot\|_{2}$ on the unit ball of $P$.

If $\Gamma$ is a countable group, then $L \Gamma$ has property (T) if and only if the group $\Gamma$ has property $(\mathrm{T})$ in the usual sense.

Relative property $(\mathbf{H})$ and property anti-(T). In [Po01], Section 2, property $(\mathrm{H})$ of a finite von Neumann algebra $M$ relative to a von Neumann subalgebra $P \subset M$ is introduced. We recall from [Po01] the following facts.

- If $\Gamma \curvearrowright P$ is a trace preserving action, then $P \rtimes \Gamma$ has property (H) relative to $P$ if and only if the group $\Gamma$ has the Haagerup property. Recall that a countable group $\Gamma$ has the Haagerup property if and only if there exists a sequence of positive definite functions $\varphi_{n}: \Gamma \rightarrow \mathbb{C}$ tending to 1 pointwise and such that for every $n$ the function $\varphi_{n}$ belongs to $c_{0}(\Gamma)$ (see e.g. [CCJJV]).

- If $M$ has property $(\mathrm{H})$ relative to $P \subset M$, there exists a sequence of normal subunital subtracial completely positive $P$-bimodular maps $\varphi_{n}: M \rightarrow M$ such that $\left\|\varphi_{n}(x)-x\right\|_{2} \rightarrow 0$ for every $x \in M$ and such that every $\varphi_{n}$ satisfies the following relative compactness property: if $\left(w_{k}\right)$ is a sequence of unitaries in $M$ satisfying $\left\|E_{P}\left(a w_{k} b\right)\right\|_{2} \rightarrow 0$ for all $a, b \in M$, then $\left\|\varphi_{n}\left(w_{k}\right)\right\|_{2} \rightarrow 0$ when $k \rightarrow \infty$. The converse is almost true, but we have no need to go into these technical details.

- If $M$ has property (H) relative to $P \subset M$, then $N \bar{\otimes} M$ has property (H) relative to $N \bar{\otimes} P$ for every finite von Neumann algebra $N$.

The following lemma is essentially contained in [Po01], Theorem 6.2. We provide a full proof for the convenience of the reader.

Lemma 1. Let $(M, \tau)$ be a tracial von Neumann algebra and $P_{1} \subset P \subset M$ von Neumann subalgebras. Assume that $P$ has property $(\mathrm{H})$ relative to $P_{1}$.

If $M_{0} \subset p M p$ is a von Neumann subalgebra such that $M_{0}$ has property (T) and $M_{0} \prec_{M} P$, then $M_{0} \prec_{M} P_{1}$.

Proof. Assume that $M_{0} \nprec_{M} P_{1}$. Since $M_{0} \prec_{M} P$, by [Va07], Remark 3.8, we find non-zero projections $p_{0} \in M_{0}, q \in P$, a non-zero partial isometry $v \in p_{0} M q$ and a normal unital $*$-homomorphism $\theta: p_{0} M_{0} p_{0} \rightarrow q P q$ such that $x v=v \theta(x)$ for all $x \in p_{0} M_{0} p_{0}$ and such that $\theta\left(p_{0} M_{0} p_{0}\right) \nprec_{P} P_{1}$.

Since $P$ has property $(\mathrm{H})$ relative to $P_{1}$, we can take a sequence $\varphi_{n}: P \rightarrow P$ of normal subunital subtracial completely positive maps such that $\left\|\varphi_{n}(x)-x\right\|_{2} \rightarrow 0$ for all $x \in P$ and such that every $\varphi_{n}$ satisfies the relative compactness property explained above. Since $\theta\left(p_{0} M_{0} p_{0}\right)$ has property (T), take $n$ such that $\left\|\varphi_{n}(w)-w\right\|_{2} \leq\|q\|_{2} / 2$ for all unitaries $w \in \theta\left(p_{0} M_{0} p_{0}\right)$. Since $\theta\left(p_{0} M_{0} p_{0}\right) \nprec_{P} P_{1}$, take a sequence of unitaries $w_{k} \in \theta\left(p_{0} M_{0} p_{0}\right)$ such that $\left\|E_{P_{1}}\left(a w_{k} b\right)\right\|_{2} \rightarrow 0$ for all $a, b \in P$. By the 
relative compactness of $\varphi_{n}$, it follows that $\left\|\varphi_{n}\left(w_{k}\right)\right\|_{2} \rightarrow 0$ when $k \rightarrow \infty$. So, for $k$ large enough, we have $\left\|\varphi_{n}\left(w_{k}\right)\right\|_{2}<\|q\|_{2} / 2$. It follows that

$$
\|q\|_{2}=\left\|w_{k}\right\|_{2} \leq\left\|\varphi_{n}\left(w_{k}\right)\right\|_{2}+\left\|\varphi_{n}\left(w_{k}\right)-w_{k}\right\|_{2}<\|q\|_{2},
$$

which is absurd.

We say that a finite von Neumann algebra $P$ is anti-(T) if there exist von Neumann subalgebras $\mathbb{C} 1=P_{0} \subset P_{1} \subset \cdots \subset P_{n}=P$ such that for all $i=1, \ldots, n$, the von Neumann algebra $P_{i}$ has property $(\mathrm{H})$ relative to $P_{i-1}$. Repeatedly applying Lemma 1, an anti-(T) von Neumann algebra cannot contain a diffuse von Neumann subalgebra with property $(\mathrm{T})$.

We say that a countable group $\Sigma$ is anti-(T) if there exist subgroups $\{e\}=\Sigma_{0}<$ $\Sigma_{1}<\cdots<\Sigma_{n}=\Sigma$ such that for all $i=1, \ldots, n, \Sigma_{i-1}$ is normal in $\Sigma_{i}$ and $\Sigma_{i} / \Sigma_{i-1}$ has the Haagerup property. If $\Sigma$ is anti-(T), then the group von Neumann algebra $\mathrm{L} \Sigma$ is anti-(T) as well. An anti-(T) group cannot contain an infinite subgroup with property (T). Nevertheless, $\operatorname{SL}(2, \mathbb{Z}) \ltimes \mathbb{Z}^{2}$ is an anti-(T) group (since $\operatorname{SL}(2, \mathbb{Z})$ has the Haagerup property and $\mathbb{Z}^{2}$ is amenable) which contains an infinite subgroup with the relative property $(\mathrm{T})$, namely $\mathbb{Z}^{2}$. This explains why our new transfer of rigidity lemma (see Lemma 4 ) requires property $(\mathrm{T})$ rather than relative property $(\mathrm{T})$.

\section{Transfer of rigidity and $W^{*}$-superrigidity}

We say that free ergodic pmp actions $\Gamma \curvearrowright(X, \mu)$ and $\Lambda \curvearrowright(Y, \eta)$ are

- $\mathrm{W}^{*}$-equivalent, if $\mathrm{L}^{\infty}(X) \rtimes \Gamma \cong \mathrm{L}^{\infty}(Y) \rtimes \Lambda$,

- orbit equivalent, if the orbit equivalence relations $\mathcal{R}(\Gamma \curvearrowright X)$ and $\mathcal{R}(\Lambda \curvearrowright Y)$ are isomorphic,

- conjugate, if there exists an isomorphism of probability spaces $\Delta: X \rightarrow Y$ and an isomorphism of groups $\delta: \Gamma \rightarrow \Lambda$ such that $\Delta(g \cdot x)=\delta(g) \cdot \Delta(x)$ almost everywhere.

Following [PV09], Definition 6.1, a free ergodic pmp action $\Gamma \curvearrowright(X, \mu)$ is called $W^{*}$-superrigid if the following property holds. If $\Lambda \curvearrowright(Y, \eta)$ is an arbitrary free ergodic pmp action and $\pi: \mathrm{L}^{\infty}(X) \rtimes \Gamma \rightarrow \mathrm{L}^{\infty}(Y) \rtimes \Lambda$ is a $\mathrm{W}^{*}$-equivalence, then the actions $\Gamma \curvearrowright X$ and $\Lambda \curvearrowright Y$ are conjugate through $\Delta: X \rightarrow Y, \delta: \Gamma \rightarrow \Lambda$ and up to unitary conjugacy $\pi$ is of the form

$$
\pi\left(a u_{g}\right)=\Delta_{*}\left(a \omega_{g}\right) u_{\delta(g)} \quad \text { for all } a \in \mathrm{L}^{\infty}(X), g \in \Gamma,
$$

where $\left(\omega_{g}\right) \in \mathrm{Z}^{1}(\Gamma \curvearrowright X)$ is a $\mathbb{T}$-valued 1-cocycle for the action $\Gamma \curvearrowright X$.

Slightly more natural than $\mathrm{W}^{*}$-superrigidity is the notion of stable $W^{*}$-superrigidity where possible finite index issues are correctly taken into account. A stable isomorphism between $\mathrm{II}_{1}$ factors $M$ and $N$ is an isomorphism between $M$ and an 
amplification $N^{t}$. This leads to the notion of stable $W^{*}$-equivalence between free ergodic pmp actions. Similarly one defines stable orbit equivalence. Finally, a stable conjugacy between two free ergodic pmp actions $\Gamma \curvearrowright(X, \mu)$ and $\Lambda \curvearrowright(Y, \eta)$ is a conjugacy between the actions $\frac{\Gamma_{0}}{G} \curvearrowright \frac{X_{0}}{G}$ and $\frac{\Lambda_{0}}{H} \curvearrowright \frac{Y_{0}}{H}$ where $\Gamma \curvearrowright X, \Lambda \curvearrowright Y$ are induced $^{1}$ from $\Gamma_{0} \curvearrowright X_{0}, \Lambda_{0} \curvearrowright Y_{0}$ and where $G \triangleleft \Gamma_{0}, H \triangleleft \Lambda_{0}$ are finite normal subgroups.

Definition 2 ([PV09], Definition 6.4). A free ergodic pmp action $\Gamma \curvearrowright(X, \mu)$ is said to be stably $W^{*}$-superrigid if the following holds. Whenever $\pi$ is a stable $\mathrm{W}^{*}$ equivalence between $\Gamma \curvearrowright(X, \mu)$ and an arbitrary free ergodic pmp action $\Lambda \curvearrowright$ $(Y, \eta)$, the actions are stably conjugate and $\pi$ equals the composition of

- the canonical stable $\mathrm{W}^{*}$-equivalence given by the stable conjugacy,

- the automorphism of $\mathrm{L}^{\infty}(X) \rtimes \Gamma$ given by an element of $\mathrm{Z}^{1}(\Gamma \curvearrowright X)$,

- an inner automorphism of $\mathrm{L}^{\infty}(X) \rtimes \Gamma$.

Let $\Gamma \curvearrowright(X, \mu)$ be stably $\mathrm{W}^{*}$-superrigid. If moreover $\Gamma$ has no finite normal subgroups and if finite index subgroups of $\Gamma$ still act ergodically on $(X, \mu)$, then $\Gamma \curvearrowright(X, \mu)$ is $\mathrm{W}^{*}$-superrigid in the sense explained above.

The following is the main result in this section.

Theorem 3. Denote by $\Sigma<\operatorname{SL}(3, \mathbb{Z})$ the subgroup of matrices $g$ such that $g_{31}=$ $g_{32}=0$. Put $\Gamma=\operatorname{SL}(3, \mathbb{Z}) *_{\Sigma} \operatorname{SL}(3, \mathbb{Z})$. Every free ergodic pmp action $\Gamma \curvearrowright(X, \mu)$ is stably $W^{*}$-superrigid. In particular all a-periodic ${ }^{2}$ free ergodic pmp actions of $\Gamma$ are $W^{*}$-superrigid.

More generally, if $k \geq 1$ and $n_{1}, \ldots, n_{k} \in\{1,2\}$, the same conclusion holds for the group $\Gamma=\operatorname{PSL}(n, \mathbb{Z}) *_{\Sigma} \operatorname{PSL}(n, \mathbb{Z})$ where $n=2+n_{1}+\cdots+n_{k}$ and $\Sigma$ is the image in $\operatorname{PSL}(n, \mathbb{Z})$ of

$$
\operatorname{SL}(n, \mathbb{Z}) \cap\left(\begin{array}{cccc}
\mathrm{GL}(2, \mathbb{Z}) & * & \cdots & * \\
0 & \mathrm{GL}\left(n_{1}, \mathbb{Z}\right) & \cdots & * \\
\vdots & \vdots & \ddots & \vdots \\
0 & 0 & \cdots & \mathrm{GL}\left(n_{k}, \mathbb{Z}\right)
\end{array}\right)
$$

Proof. The theorem is a direct consequence of Kida's [Ki09], Theorem 9.11, and the

\footnotetext{
${ }^{1} \mathrm{~A}$ free ergodic pmp action $\Gamma \curvearrowright(X, \mu)$ is said to be induced from $\Gamma_{0} \curvearrowright X_{0}$ if $\Gamma_{0}<\Gamma$ is a finite index subgroup and $X_{0} \subset X$ is a non-negligible $\Gamma_{0}$-invariant subset such that $\mu\left(X_{0} \cap g \cdot X_{0}\right)=0$ for all $g \in \Gamma-\Gamma_{0}$.

${ }^{2} \mathrm{~A}$ free ergodic pmp action is called a-periodic if it is not induced from a finite index subgroup, i.e., if finite index subgroups still act ergodically.
} 
uniqueness of group measure space Cartan Theorem 5 below. Let

$$
P=\left(\begin{array}{cccc}
1 & * & \cdots & * \\
0 & 1 & \cdots & * \\
\vdots & \vdots & \ddots & \vdots \\
0 & 0 & \cdots & 1
\end{array}\right)
$$

and

$$
G=\operatorname{SL}(n, \mathbb{Z}) \cap\left(\begin{array}{cccc}
\mathrm{GL}(2, \mathbb{Z}) & 0 & \cdots & 0 \\
0 & \mathrm{GL}\left(n_{1}, \mathbb{Z}\right) & \cdots & 0 \\
\vdots & \vdots & \ddots & \vdots \\
0 & 0 & \cdots & \mathrm{GL}\left(n_{k}, \mathbb{Z}\right)
\end{array}\right)
$$

and denote by $\bar{P}$ (resp. $\bar{G}$ ) the image of $P$ (resp. $G$ ) in $\operatorname{PSL}(n, \mathbb{Z})$. We have that $\bar{P}$ is amenable and normal in $\Sigma$ and $\bar{G} \cong \Sigma / \bar{P}$ has the Haagerup property. This shows that $\Sigma$ is anti-(T). Therefore, if $\Gamma \curvearrowright(X, \mu)$ is an arbitrary free ergodic pmp action, Theorem 5 says that every stable $\mathrm{W}^{*}$-equivalence between $\Gamma \curvearrowright(X, \mu)$ and an arbitrary $\Lambda \curvearrowright(Y, \eta)$ comes from a stable orbit equivalence of the actions. Kida showed in [Ki09], Theorem 9.11, that $\Gamma$ is coupling rigid with respect to the abstract commensurator $^{3} \operatorname{Comm}(\Gamma)$. Since $\Gamma$ is icc and $\operatorname{Comm}(\Gamma)$ is countable, this precisely means that every stable orbit equivalence comes from a stable conjugacy, cf. [Ki09], Proposition 3.11.

The $\mathrm{W}^{*}$-superrigidity in Theorem 3 arises as the combination of Kida's OE superrigidity and the following uniqueness result for group measure space Cartan subalgebras. We first need a new transfer of rigidity lemma (cf. [PV09], Lemma 3.1).

Lemma 4. Let $M$ be a $\mathrm{II}_{1}$ factor and $\varphi_{n}: M \rightarrow M$ a sequence of normal subunital subtracial completely positive maps. Assume that $P, M_{0} \subset M$ are von Neumann subalgebras such that $P$ is anti-(T) and such that $M_{0}$ is diffuse and has property (T).

Let $p \in M$ be a projection and $p M p=Q \rtimes \Lambda$ any crossed product decomposition with $Q$ being anti-(T). Denote by $\left(v_{s}\right)_{s \in \Lambda}$ the corresponding canonical unitaries.

For every $\varepsilon>0$, there exists $n$ and a sequence $\left(s_{k}\right)_{k \in \mathbb{N}}$ in $\Lambda$ such that

(1) $\left\|\varphi_{n}\left(v_{s_{k}}\right)-v_{s_{k}}\right\|_{2} \leq \varepsilon$ for all $k \in \mathbb{N}$,

(2) for all $a, b \in M$ we have $\left\|E_{P}\left(a v_{s_{k}} b\right)\right\|_{2} \rightarrow 0$ when $k \rightarrow \infty$.

Proof. Since $M$ is a $\mathrm{II}_{1}$ factor and $M_{0}$ is diffuse, we may assume that $p \in M_{0}$. Write $N=Q \rtimes \Lambda$ and denote by $\Delta: N \rightarrow N \bar{\otimes} N$ the normal $*$-homomorphism given

\footnotetext{
${ }^{3}$ Given a group $\Gamma$ the abstract commensurator $\operatorname{Comm}(\Gamma)$ is defined as the group of all isomorphisms $\delta: \Gamma_{1} \rightarrow \Gamma_{2}$ between finite index subgroups $\Gamma_{1}, \Gamma_{2}<\Gamma$, identifying two such isomorphisms when they coincide on a finite index subgroup. Inner conjugacy provides a homomorphism from $\Gamma$ to $\operatorname{Comm}(\Gamma)$, which is injective if and only if $\Gamma$ is icc.
} 
by $\Delta\left(a v_{s}\right)=a v_{s} \otimes v_{s}$ for all $a \in Q$ and $s \in \Lambda$. Normalize the trace $\tau$ on $M$ such that $\tau(p)=1$.

Since $Q$ is anti-(T), Lemma 1 implies that $p M_{0} p \nprec_{N} Q$. Let $\left(w_{n}\right)$ be a sequence of unitaries in $p M_{0} p$ such that $\left\|E_{Q}\left(a w_{n} b\right)\right\|_{2} \rightarrow 0$ for all $a, b \in N$. It follows that $\Delta\left(w_{n}\right)$ is a sequence of unitaries in $N \bar{\otimes} N$ satisfying

$$
\left\|(\mathrm{id} \otimes \tau)\left(a \Delta\left(w_{n}\right) b\right)\right\|_{2} \rightarrow 0 \quad \text { for all } a, b \in N \bar{\otimes} N .
$$

Indeed, it suffices to check the convergence for $a=1 \otimes c v_{s}$ and $b=1 \otimes d v_{t}$, where $c, d \in Q$ and $s, t \in \Lambda$. We have $\left\|(\mathrm{id} \otimes \tau)\left(\left(1 \otimes c v_{s}\right) \Delta\left(w_{n}\right)\left(1 \otimes d v_{t}\right)\right)\right\|_{2}=$ $\left|\tau\left(\sigma_{t-1}(d) c\right)\right|\left\|E_{Q}\left(w_{n} v_{t s}\right)\right\|_{2} \rightarrow 0$. So, $\Delta\left(p M_{0} p\right) \nprec_{N \bar{\otimes} N} N \otimes 1$. Since $P$ is anti-(T), Lemma 1 implies that $\Delta\left(p M_{0} p\right) \nprec_{N \bar{\otimes} M} N \bar{\otimes} P$.

Choose $\varepsilon>0$. Put $\varepsilon_{1}=\varepsilon^{2} / 4$. Since $p M_{0} p$ has property (T), take $n$ such that

$$
1-\operatorname{Re}(\tau \otimes \tau)\left(\Delta(w)^{*}\left(\mathrm{id} \otimes \varphi_{n}\right) \Delta(w)\right) \leq \varepsilon_{1}
$$

for all $w \in \mathcal{U}\left(p M_{0} p\right)$. Define

$$
\mathcal{V}:=\left\{s \in \Lambda \mid 1-\operatorname{Re} \tau\left(v_{s}^{*} \varphi_{n}\left(v_{s}\right)\right) \leq 2 \varepsilon_{1}\right\} .
$$

Note that for all $s \in \mathcal{V}$, we have $\left\|\varphi_{n}\left(v_{s}\right)-v_{s}\right\|_{2} \leq \sqrt{4 \varepsilon_{1}}=\varepsilon$. In order to prove the lemma, it suffices to show that there exists a sequence $s_{k} \in \mathcal{V}$ such that $\left\|E_{P}\left(a v_{s_{k}} b\right)\right\|_{2} \rightarrow 0$ for all $a, b \in M$. Assume the contrary. We then find a finite subset $\mathcal{F} \subset M$ and a $\delta>0$ such that

$$
\sum_{a, b \in \mathcal{F}}\left\|E_{P}\left(a v_{s} b\right)\right\|_{2}^{2} \geq \delta \quad \text { for all } s \in \mathcal{V} .
$$

We will deduce that $\Delta\left(p M_{0} p\right) \prec_{N \bar{\otimes} M} N \bar{\otimes} P$. This will be a contradiction with the statement $\Delta\left(p M_{0} p\right) \nprec_{N \bar{\otimes} M} N \bar{\otimes} P$ that we have shown at the beginning of the proof.

Let $w \in \mathcal{U}\left(p M_{0} p\right)$. Write $w=\sum_{s \in \Lambda} w_{s} v_{s}$ where $w_{s} \in Q$. Since $\tau(p)=1$, we have $\sum_{s \in \Lambda}\left\|w_{s}\right\|_{2}^{2}=1$. Therefore,

$$
\begin{aligned}
\varepsilon_{1} & \geq 1-\operatorname{Re}(\tau \otimes \tau)\left(\Delta(w)^{*}\left(\operatorname{id} \otimes \varphi_{n}\right) \Delta(w)\right) \\
& =\sum_{s \in \Lambda}\left\|w_{s}\right\|_{2}^{2}\left(1-\operatorname{Re} \tau\left(v_{s}^{*} \varphi_{n}\left(v_{s}\right)\right)\right) \\
& \geq \sum_{s \in \Lambda-\mathcal{V}}\left\|w_{s}\right\|_{2}^{2}\left(1-\operatorname{Re} \tau\left(v_{s}^{*} \varphi_{n}\left(v_{s}\right)\right)\right) \\
& \geq \sum_{s \in \Lambda-\mathcal{V}}\left\|w_{s}\right\|_{2}^{2} 2 \varepsilon_{1} .
\end{aligned}
$$

We conclude that for all $w \in \mathcal{U}\left(p M_{0} p\right)$ we have

$$
\sum_{s \in \Lambda-\mathcal{V}}\left\|w_{s}\right\|_{2}^{2} \leq \frac{1}{2}
$$


implying that

$$
\sum_{s \in \mathcal{V}}\left\|w_{s}\right\|_{2}^{2} \geq \frac{1}{2}
$$

for all $w \in \mathcal{U}\left(p M_{0} p\right)$.

It follows that for all $w \in U\left(p M_{0} p\right)$

$$
\begin{aligned}
\sum_{a, b \in \mathcal{F}}\left\|E_{N \bar{\otimes} P}((1 \otimes a) \Delta(w)(1 \otimes b))\right\|_{2}^{2} & =\sum_{a, b \in \mathcal{F}, s \in \Lambda}\left\|w_{s}\right\|_{2}^{2}\left\|E_{P}\left(a v_{s} b\right)\right\|_{2}^{2} \\
& \geq \sum_{a, b \in \mathcal{F}, s \in \mathcal{V}}\left\|w_{s}\right\|_{2}^{2}\left\|E_{P}\left(a v_{s} b\right)\right\|_{2}^{2} \\
& \geq \sum_{s \in \mathcal{V}}\left\|w_{s}\right\|_{2}^{2} \delta \\
& \geq \frac{\delta}{2} .
\end{aligned}
$$

This means that $\Delta\left(p M_{0} p\right) \prec_{N \bar{\otimes} M} N \bar{\otimes} P$. We have reached the desired contradiction.

We are ready to formulate and prove our uniqueness of group measure space Cartan theorem. We use the notation $\mathrm{D}_{n}(\mathbb{C}) \subset \mathrm{M}_{n}(\mathbb{C})$ to denote the subalgebra of diagonal matrices.

Theorem 5. Let $\Gamma=\Gamma_{1} *_{\Sigma} \Gamma_{2}$ be an amalgamated free product satisfying the following conditions: $\Gamma_{1}$ admits an infinite subgroup with property $(\mathrm{T}), \Sigma$ is anti(T) and $\Gamma_{2} \neq \Sigma$. Assume moreover that there exist $g_{1}, \ldots, g_{n} \in \Gamma$ such that $\bigcap_{i=1}^{n} g_{i} \Sigma g_{i}^{-1}$ is finite. Let $\Gamma \curvearrowright(X, \mu)$ be any free ergodic pmp action and denote $M=\mathrm{L}^{\infty}(X) \rtimes \Gamma$.

Whenever $\Lambda \curvearrowright(Y, \eta)$ is a free ergodic pmp action, $p \in \mathrm{M}_{n}(\mathbb{C}) \otimes M$ is a projection and

$$
\pi: \mathrm{L}^{\infty}(Y) \rtimes \Lambda \rightarrow p\left(\mathrm{M}_{n}(\mathbb{C}) \otimes M\right) p
$$

is a $*$-isomorphism, there exists a projection $q \in \mathrm{D}_{n}(\mathbb{C}) \otimes \mathrm{L}^{\infty}(X)$ and a unitary $u \in q\left(\mathbf{M}_{n}(\mathbb{C}) \otimes M\right) p$ such that

$$
\pi\left(\mathrm{L}^{\infty}(Y)\right)=u^{*}\left(\mathrm{D}_{n}(\mathbb{C}) \otimes \mathrm{L}^{\infty}(X)\right) u .
$$

Proof. Write $A=\mathrm{M}_{n}(\mathbb{C}) \otimes \mathrm{L}^{\infty}(X)$ and $N=A \rtimes \Gamma$. Put $B=\mathrm{L}^{\infty}(Y)$. We first prove that $\pi(B) \prec_{N} A \rtimes \Sigma$. Denote by $|g|$ the length of $g \in \Gamma_{1} *_{\Sigma} \Gamma_{2}$ as a reduced word. Denote for $0<\rho<1$ by $\mathrm{m}_{\rho}$ the corresponding completely positive maps on $N$ given by $\mathrm{m}_{\rho}\left(a u_{g}\right)=\rho^{|g|} a u_{g}$ for all $a \in A, g \in \Gamma$. When $\rho \rightarrow 1$, we have $\mathrm{m}_{\rho} \rightarrow$ id pointwise in $\|\cdot\|_{2}$.

Write $\varepsilon=\tau(p) / 5000$ and $P=A \rtimes \Sigma$. Note that $P$ is anti-(T). By Lemma 4 take $0<\rho_{1}<1$ and a sequence $\left(s_{k}\right)$ in $\Lambda$ satisfying $\left\|m_{\rho_{1}}\left(v_{s_{k}}\right)-v_{s_{k}}\right\|_{2} \leq \varepsilon$ for all 
$k$ and $\left\|E_{P}\left(x v_{s_{k}} y\right)\right\|_{2} \rightarrow 0$ for all $x, y \in N$. By [PV09], Lemma 5.7, there exists a $0<\rho<1$ and a $\delta>0$ such that $\tau\left(w^{*} \mathrm{~m}_{\rho}(w)\right) \geq \delta$ for all $w \in \mathcal{U}(\pi(B))$. By [PV09], Theorem 5.4, and because $\pi(B)$ is regular in $p N p$, it follows that $\pi(B) \prec_{N} P$.

So we have shown that $\pi(B) \prec_{N} A \rtimes \Sigma$. By Proposition 8 below and since we have $g_{1}, \ldots, g_{n} \in \Gamma$ such that $\bigcap_{i=1}^{n} g_{i} \Sigma g_{i}^{-1}$ is finite, it follows that $\pi(B) \prec_{N} A$. The theorem now follows from [Po01], Theorem A.1.

\section{An embedding result, strengthening [PV06], Theorem 6.16}

Assume that $(A, \tau)$ is a tracial von Neumann algebra and that $\Gamma \stackrel{\sigma}{\curvearrowright}(A, \tau)$ is a trace preserving action. We do not assume that $\sigma$ is properly outer or that $\sigma$ is ergodic. Let $M=A \rtimes \Gamma$.

Whenever $\Lambda<\Gamma$ is a subgroup, consider the basic construction $\left\langle M, e_{A \rtimes \Lambda}\right\rangle$. By definition $\left\langle M, e_{A \rtimes \Lambda}\right\rangle$ consists of those operators on $\mathrm{L}^{2}(M)$ that commute with the right module action of $A \rtimes \Lambda$. The basic construction comes with a canonical operator valued weight $T_{\Lambda}$ from $\left\langle M, e_{A \rtimes \Lambda}\right\rangle^{+}$to the extended positive part of $M$. For all $x, y \in$ $M$, the element $x e_{A \rtimes \Lambda} y$ is integrable with respect to $T_{\Lambda}$ and $T_{\Lambda}\left(x e_{A \rtimes \Lambda} y\right)=x y$. Choose $g_{i} \in \Gamma$ such that $\Gamma=\bigsqcup_{i} g_{i} \Lambda=\bigsqcup_{i} \Lambda g_{i}^{-1}$. Denoting by $\rho_{g}$ the right multiplication operator by $u_{g}^{*}$ on $\mathrm{L}^{2}(M)$, one checks that $\sum_{i} \rho_{g_{i}} e_{A \rtimes \Lambda} \rho_{g_{i}}^{*}=1$, whence

$$
T_{\Lambda}(x)=\sum_{i} \rho_{g_{i}} x \rho_{g_{i}}^{*} \quad \text { for all } x \in\left\langle M, e_{A \rtimes \Lambda}\right\rangle^{+} .
$$

The canonical semi-finite trace $\operatorname{Tr}_{\Lambda}$ on $\left\langle M, e_{A \rtimes \Lambda}\right\rangle$ is given as the composition of $T_{\Lambda}$ and the trace $\tau$ on $M$.

Assume that $p \in M$ is a projection and $B \subset p M p$ is a quasi-regular subalgebra (see [Po01], 1.4.2). Recall that this means that the quasi-normalizer of $B$ inside $p M p$ is weakly dense in $p M p$. Obviously, regular subalgebras $B \subset p M p$ or, even more specifically, Cartan subalgebras $B \subset p M p$ are quasi-regular.

Given a subgroup $\Lambda<\Gamma$, let $H \subset p \mathrm{~L}^{2}(M)$ be the closed linear span of all $B-(A \rtimes \Lambda)$-subbimodules of $p \mathrm{~L}^{2}(M)$ that are finitely generated as a right Hilbert $(A \rtimes \Lambda$ )-module. Since $B \subset p M p$ is quasi-regular, $H$ is stable by left multiplication with $p M p$. The subspace $H$ is also invariant under right multiplication by $A \rtimes \Lambda$. So $H$ is of the form $p \mathrm{~L}^{2}(M) z(\Lambda)$ for some projection $z(\Lambda) \in M \cap(A \rtimes \Lambda)^{\prime}$. We make $z(\Lambda)$ uniquely defined by requiring that $z(\Lambda)$ is smaller or equal than the central support of $p$ in $M$.

Note that by definition $z(\Lambda) \neq 0$ if and only if $B \prec_{M} A \rtimes \Lambda$.

Denote by $J: \mathrm{L}^{2}(M) \rightarrow \mathrm{L}^{2}(M)$ the adjoint operator. So, given $x \in M, J x^{*} J$ is the operator of right multiplication with $x$. Denote by supp $a$ the support projection 
of a positive operator $a$. Observe that

$$
\begin{aligned}
& p J z(\Lambda) J \\
& =\bigvee\left\{q \mid q \text { is an orthogonal projection in } B^{\prime} \cap p\left\langle M, e_{A \rtimes \Lambda}\right\rangle p\right. \\
& \left.\quad \text { satisfying } \operatorname{Tr}_{\Lambda}(q)<\infty\right\} \\
& =\bigvee\left\{q \mid q \text { is an orthogonal projection in } B^{\prime} \cap p\left\langle M, e_{A \rtimes \Lambda}\right\rangle p\right. \\
& \left.\quad \text { satisfying }\left\|T_{\Lambda}(q)\right\|<\infty\right\} \\
& =\bigvee\left\{\operatorname{supp} a \mid a \in\left(B^{\prime} \cap p\left\langle M, e_{A \rtimes \Lambda}\right\rangle p\right)^{+} \text {and }\left\|T_{\Lambda}(a)\right\|<\infty\right\} .
\end{aligned}
$$

If $a$ and $b$ are positive operators, then $\operatorname{supp}(a) \vee \operatorname{supp}(b)=\operatorname{supp}(a+b)$. So we find a sequence of elements $a_{n} \in\left(B^{\prime} \cap p\left\langle M, e_{A \rtimes \Lambda}\right\rangle p\right)^{+}$such that all $T_{\Lambda}\left(a_{n}\right)$ are bounded and $\operatorname{supp}\left(a_{n}\right) \rightarrow p J z(\Lambda) J$ strongly. Moreover, every projection $\operatorname{supp}\left(a_{n}\right)$ can be strongly approximated by a spectral projection of the form $q_{n}=\chi_{\left[\varepsilon_{n},+\infty\right)}\left(a_{n}\right)$ for $\varepsilon_{n}>0$ sufficiently small. We have $q_{n} \leq \frac{1}{\varepsilon_{n}} a_{n}$ so that $T_{\Lambda}\left(q_{n}\right)$ is bounded. Hence we find a sequence of projections $q_{n} \in B^{\prime} \cap p\left\langle M, e_{A \rtimes \Lambda}\right\rangle p$ such that $q_{n} \rightarrow p J z(\Lambda) J$ strongly and $\left\|T_{\Lambda}\left(q_{n}\right)\right\|<\infty$ for all $n$.

Note that $z\left(\Lambda_{1}\right) \leq z\left(\Lambda_{2}\right)$ when $\Lambda_{1}<\Lambda_{2}<\Gamma$. Indeed, it suffices to observe that for every $B$ - $\left(A \rtimes \Lambda_{1}\right)$-subbimodule $K \subset p \mathrm{~L}^{2}(M)$ that is finitely generated as a right Hilbert module, the closed linear span of $K\left(A \rtimes \Lambda_{2}\right)$ is a $B-\left(A \rtimes \Lambda_{2}\right)$-subbimodule of $p \mathrm{~L}^{2}(M)$ that is finitely generated as a right Hilbert module. Hence $p \mathrm{~L}^{2}(M) z\left(\Lambda_{1}\right) \subset$ $p \mathrm{~L}^{2}(M) z\left(\Lambda_{2}\right)$. Since by convention $z\left(\Lambda_{1}\right)$ and $z\left(\Lambda_{2}\right)$ are smaller or equal than the central support of $p$, we conclude that $z\left(\Lambda_{1}\right) \leq z\left(\Lambda_{2}\right)$.

By definition, $z\left(g \Lambda g^{-1}\right)=u_{g} z(\Lambda) u_{g}^{*}$ for all $g \in \Gamma, \Lambda<\Gamma$.

Proposition 6. Let $M=A \rtimes \Gamma$ for some trace preserving action of a countable group $\Gamma$ on the tracial von Neumann algebra $(A, \tau)$. Let $B \subset p M p$ be a quasi-regular von Neumann subalgebra. For every subgroup $\Lambda<\Gamma$, define as above the projection $z(\Lambda) \in M \cap(A \rtimes \Lambda)^{\prime}$ such that $p \mathrm{~L}^{2}(M) z(\Lambda)$ equals the closed linear span of all $B-(A \rtimes \Lambda)$-subbimodules of $p \mathrm{~L}^{2}(M)$ that are finitely generated as a right Hilbert module.

If $\Sigma<\Gamma$ and $\Lambda<\Gamma$ are subgroups, then the projections $z(\Sigma)$ and $z(\Lambda)$ commute and satisfy

$$
z(\Sigma \cap \Lambda)=z(\Sigma) z(\Lambda) .
$$

Proof. We use the operator valued weight $T_{\Sigma}$ as explained above. As we saw after formulae (1) we can take projections $q_{n} \in B^{\prime} \cap p\left\langle M, e_{A \rtimes \Sigma}\right\rangle p$ and $e_{n} \in B^{\prime} \cap$ $p\left\langle M, e_{A \rtimes \Lambda}\right\rangle p$ such that $q_{n} \rightarrow p J z(\Sigma) J$ and $e_{n} \rightarrow p J z(\Lambda) J$ strongly and such that for all $n \in \mathbb{N}$, we have $\left\|T_{\Sigma}\left(q_{n}\right)\right\|<\infty$ and $\left\|T_{\Lambda}\left(e_{n}\right)\right\|<\infty$. Note that $e_{n} q_{n} e_{n} \in$ $\left(B^{\prime} \cap p\left\langle M, e_{A \rtimes(\Sigma \cap \Lambda)}\right\rangle p\right)^{+}$. We claim that

$$
\left\|T_{\Sigma \cap \Lambda}\left(e_{n} q_{n} e_{n}\right)\right\|<\infty \text { for all } n \in \mathbb{N} .
$$

Fix $n \in \mathbb{N}$. Take $g_{i} \in \Gamma$ such that $\Gamma=\bigsqcup_{i} g_{i} \Lambda$. Take $h_{j} \in \Lambda$ such that $\Lambda=$ $\bigsqcup_{j} h_{j}(\Sigma \cap \Lambda)$. Note that the cosets $h_{j} \Sigma$ are disjoint and that $\Gamma=\bigsqcup_{i, j} g_{i} h_{j}(\Sigma \cap \Lambda)$. 
Because of the latter, we have

$$
T_{\Sigma \cap \Lambda}\left(e_{n} q_{n} e_{n}\right)=\sum_{i, j} \rho_{g_{i} h_{j}} e_{n} q_{n} e_{n} \rho_{g_{i} h_{j}}^{*}=\sum_{i} \rho_{g_{i}} e_{n}\left(\sum_{j} \rho_{h_{j}} q_{n} \rho_{h_{j}}^{*}\right) e_{n} \rho_{g_{i}}^{*}
$$

Because the cosets $h_{j} \Sigma$ are disjoint, we know that

$$
\sum_{j} \rho_{h_{j}} q_{n} \rho_{h_{j}}^{*} \leq \sum_{h \in \Gamma / \Sigma} \rho_{h} q_{n} \rho_{h}^{*}=T_{\Sigma}\left(q_{n}\right) \leq\left\|T_{\Sigma}\left(q_{n}\right)\right\| 1 .
$$

Therefore,

$$
T_{\Sigma \cap \Lambda}\left(e_{n} q_{n} e_{n}\right) \leq\left\|T_{\Sigma}\left(q_{n}\right)\right\| \sum_{i} \rho_{g_{i}} e_{n} \rho_{g_{i}}^{*}=\left\|T_{\Sigma}\left(q_{n}\right)\right\| T_{\Lambda}\left(e_{n}\right) .
$$

So claim (2) is proven.

Since $e_{n} q_{n} e_{n} \in\left(B^{\prime} \cap p\left\langle M, e_{A \rtimes(\Sigma \cap \Lambda)}\right\rangle p\right)^{+}$claim (2) implies that

$$
\operatorname{supp}\left(e_{n} q_{n} e_{n}\right) \leq p J z(\Sigma \cap \Lambda) J \quad \text { for all } n \in \mathbb{N} \text {. }
$$

Hence, $\operatorname{Ran}\left(e_{n} q_{n}\right) \subset p \mathrm{~L}^{2}(M) z(\Sigma \cap \Lambda)$ for all $n$. Since $e_{n} \rightarrow p J z(\Lambda) J$ and $q_{n} \rightarrow p J z(\Sigma) J$ strongly and since $z(\Lambda)$ and $z(\Sigma)$ are chosen below the central support of $p$, it follows that

$$
\operatorname{Ran}(z(\Lambda) z(\Sigma)) \subset \operatorname{Ran} z(\Sigma \cap \Lambda)
$$

Since $z(\Sigma \cap \Lambda) \leq z(\Sigma)$ and $z(\Sigma \cap \Lambda) \leq z(\Lambda)$, we get the chain of inclusions

$\operatorname{Ran} z(\Lambda) \cap \operatorname{Ran} z(\Sigma) \subset \operatorname{Ran}(z(\Lambda) z(\Sigma)) \subset \operatorname{Ran} z(\Sigma \cap \Lambda) \subset \operatorname{Ran} z(\Lambda) \cap \operatorname{Ran} z(\Sigma)$

So all these inclusions are equalities. This means that $z(\Lambda)$ and $z(\Sigma)$ are commuting projections and $z(\Sigma \cap \Lambda)=z(\Sigma) z(\Lambda)$.

As an immediate corollary we find the following generalization of [PV06], Theorem 6.16.

Corollary 7. Let $\Gamma \curvearrowright A$ be a trace preserving action, $M=A \rtimes \Gamma, \Sigma<\Gamma a$ subgroup and $B \subset p M p$ a quasi-regular von Neumann subalgebra. If $z(\Sigma)$ equals the central support of $p$, then the same is true for $z\left(g_{1} \Sigma g_{1}^{-1} \cap \cdots \cap g_{n} \Sigma g_{n}^{-1}\right)$ and in particular

$$
B \prec_{M} A \rtimes\left(g_{1} \Sigma g_{1}^{-1} \cap \cdots \cap g_{n} \Sigma g_{n}^{-1}\right)
$$

for all $g_{1}, \ldots, g_{n} \in \Gamma$ 


\section{Application to the proofs of Theorem 5, [PV09], Theorems 5.2 and 1.4, and [FV10], Theorem 4.1}

In the setup of Theorem 5 and [PV09], Theorems 5.2 and 1.4, we know that $\Gamma=$ $\Gamma_{1} *_{\Sigma} \Gamma_{2}$ is an amalgamated free product and we know that $A \rtimes \Gamma$ is a factor. We prove that in such a situation, whenever $B \subset p(A \rtimes \Gamma) p$ is a quasi-regular subalgebra, $z(\Sigma)$ can only take the values 0 or 1 . A similar statement is true when $\Gamma=\operatorname{HNN}(H, \Sigma, \theta)$ is an HNN extension ${ }^{4}$ of a countable group $H$ with a subgroup $\Sigma<H$ and an injective group homomorphism $\theta: \Sigma \rightarrow H$.

The precise formulation goes as follows.

Proposition 8. Let $\Gamma$ either be an amalgamated free product $\Gamma=\Gamma_{1} *_{\Sigma} \Gamma_{2}$ with $\Gamma_{1} \neq \Sigma \neq \Gamma_{2}$ or an arbitrary HNN extension $\Gamma=\operatorname{HNN}(H, \Sigma, \theta)$. Let $(A, \tau)$ be a tracial von Neumann algebra and $\Gamma \curvearrowright A$ a trace preserving action. Put $M=A \rtimes \Gamma$ and let $B \subset p M p$ be a quasi-regular von Neumann subalgebra. As above we define for every subgroup $\Lambda<\Gamma$, the projection $z(\Lambda) \in M \cap(A \rtimes \Lambda)^{\prime}$ such that $p \mathrm{~L}^{2}(M) z(\Lambda)$ is the closed linear span of all $B-(A \rtimes \Lambda)$-subbimodules of $p \mathrm{~L}^{2}(M)$ that are finitely generated as a right Hilbert module.

The projection $z(\Sigma)$ belongs to the center of $M$. So, if $M$ is a factor and if $B \prec_{M} A \rtimes \Sigma$, then $z(\Sigma)=1$ and

$$
B \prec_{M} A \rtimes\left(g_{1} \Sigma g_{1}^{-1} \cap \cdots \cap g_{n} \Sigma g_{n}^{-1}\right)
$$

for all $g_{1}, \ldots, g_{n} \in \Gamma$.

Proof. Assume first that $\Gamma=\Gamma_{1} *_{\Sigma} \Gamma_{2}$ is a non-trivial amalgamated free product. We use Proposition 6 to prove that $z(\Sigma)=z\left(\Gamma_{1}\right)$. Once this is proven, by symmetry also $z(\Sigma)=z\left(\Gamma_{2}\right)$. But then $z(\Sigma)$ commutes with both $A \rtimes \Gamma_{1}$ and $A \rtimes \Gamma_{2}$, so that $z(\Sigma)$ belongs to the center of $M$.

Put $z_{1}=z\left(\Gamma_{1}\right)$. Define $S \subset \Gamma$ as the set of elements $g \in \Gamma$ that admit a reduced expression starting with a letter from $\Gamma_{2}-\Sigma$. Whenever $g \in S$, we have $g \Gamma_{1} g^{-1} \cap \Gamma_{1} \subset \Sigma$. It follows from Proposition 6 that the projections $z_{1}=z\left(\Gamma_{1}\right)$ and $u_{g} z_{1} u_{g}^{*}=z\left(g \Gamma_{1} g^{-1}\right)$ commute and that

$$
z(\Sigma) \geq z\left(g \Gamma_{1} g^{-1} \cap \Gamma_{1}\right)=u_{g} z_{1} u_{g}^{*} z_{1} \quad \text { for all } g \in S .
$$

We claim that

$$
z_{1}=\bigvee_{g \in S} u_{g} z_{1} u_{g}^{*} z_{1}
$$

To prove this claim, put

$$
q=z_{1}-\bigvee_{g \in S} u_{g} z_{1} u_{g}^{*} z_{1}
$$

\footnotetext{
${ }^{4}$ The HNN extension $\operatorname{HNN}(H, \Sigma, \theta)$ is generated by a copy of $H$ and an element $t$, called stable letter, subject to the relation $t \sigma t^{-1}=\theta(\sigma)$ for all $\sigma \in \Sigma$.
} 
Whenever $g \in S$, we have

$$
q u_{g} q u_{g}^{*}=q z_{1} u_{g} z_{1} q u_{g}^{*}=q z_{1} u_{g} z_{1} u_{g}^{*} u_{g} q u_{g}^{*}=q u_{g} z_{1} u_{g}^{*} z_{1} u_{g} q u_{g}^{*}=0 .
$$

Take $a \in \Gamma_{1}-\Sigma$ and $b \in \Gamma_{2}-\Sigma$ and put $u_{n}=u_{(b a)^{n}}$. It follows that the projections $u_{n} q u_{n}^{*}, n \in \mathbb{N}$, are mutually orthogonal. Indeed, if $n<m$, we have

$$
u_{n} q u_{n}^{*} u_{m} q u_{m}^{*}=u_{n}\left(q u_{m-n} q u_{m-n}^{*}\right) u_{n}^{*}=0
$$

because $(b a)^{m-n} \in S$. Since $\tau$ is a finite trace on $M$, it follows that $\tau(q)=0$ and hence, $q=0$. This proves the claim. In combination with (4) it follows that $z(\Sigma) \geq z_{1}$. Hence, $z(\Sigma)=z_{1}$.

Next assume that $\Gamma=\operatorname{HNN}(H, \Sigma, \theta)$. Denote by $t \in \Gamma$ the stable letter. For every $n \geq 1$, one has $t^{-n} H t^{n} \cap H \subset \Sigma$. The same argument as in the case of amalgamated free products shows that $z(\Sigma)=z(H)$. We also have $\Sigma \subset t^{-1} H t$. Hence $z(\Sigma) \leq z\left(t^{-1} H t\right)$. Since $z(H)=z(\Sigma)$ and $z\left(t^{-1} H t\right)=u_{t}^{*} z(H) u_{t}$, we conclude that $z(H) \leq u_{t}^{*} z(H) u_{t}$. The left and right hand side have the same trace and hence must be equal. It follows that $z(\Sigma)$ commutes with $u_{t}$. Since $z(\Sigma)$ already commutes with $A \rtimes H$, it follows that $z(\Sigma)$ belongs to the center of $M$.

Finally consider the special case where $M$ is a factor and $B \prec_{M} A \rtimes \Sigma$. The latter precisely means that $z(\Sigma) \neq 0$. Since $z(\Sigma)$ is a projection in the center of $M$, it follows that $z(\Sigma)=1$. Then also $z\left(g \Sigma g^{-1}\right)=1$ for all $g \in \Gamma$. By Proposition 6 , we get that

$$
z\left(g_{1} \Sigma g_{1}^{-1} \cap \cdots \cap g_{n} \Sigma g_{n}^{-1}\right)=1
$$

for all $g_{1}, \ldots, g_{n} \in \Gamma$. In particular,

$$
B \prec_{M} A \rtimes\left(g_{1} \Sigma g_{1}^{-1} \cap \cdots \cap g_{n} \Sigma g_{n}^{-1}\right)
$$

for all $g_{1}, \ldots, g_{n} \in \Gamma$.

\section{References}

[CCJJV] P.-A. Cherix, M. Cowling, P. Jolissaint, P. Julg, and A. Valette, Groups with the Haagerup property. Progr. Math. 197, Birkhäuser Verlag, Basel 2001. Zbl 1030.43002 MR 1852148

[CJ85] A. Connes and V. Jones, Property $T$ for von Neumann algebras. Bull. London Math. Soc. 17 (1985), 57-62. Zbl 1190.46047 MR 766450

[FV10] P. Fima and S. Vaes, HNN extensions and unique group measure space decomposition of $\mathrm{II}_{1}$ factors. Trans. Amer. Math. Soc. 364 (2012), 2601-2617. Zbl 1251.46032 MR 2888221

[Ki09] Y. Kida, Rigidity of amalgamated free products in measure equivalence. J. Topol. 4 (2011), 687-735. Zbl 05955244 MR 2832574 
[Po01] S. Popa, On a class of type $\mathrm{II}_{1}$ factors with Betti numbers invariants. Ann. of Math. (2) 163 (2006), 809-899. Zbl 1120.46045 MR 2215135

[Po03] S. Popa, Strong rigidity of $\mathrm{II}_{1}$ factors arising from malleable actions of $w$-rigid groups, I. Invent. Math. 165 (2006), 369-408. Zbl 1120.46043 MR 2231961

[PV06] S. Popa and S. Vaes, Strong rigidity of generalized Bernoulli actions and computations of their symmetry groups. Adv. Math. 217 (2008), 833-872. Zbl 1137.37003 MR 2370283

[PV09] S. Popa and S. Vaes, Group measure space decomposition of $\mathrm{II}_{1}$ factors and $W^{*}$ superrigidity. Invent. Math. 182 (2010), 371-417. Zbl 1238.46052 MR 2729271

[Va07] S. Vaes, Explicit computations of all finite index bimodules for a family of $\mathrm{II}_{1}$ factors. Ann. Sci. Éc. Norm. Supér. (4) 41 (2008), 743-788. Zbl 1194.46086 MR 2504433

Received July 7, 2011; revised October 30, 2011

C. Houdayer, CNRS-ENS Lyon, UMPA UMR 5669, 69364 Lyon Cedex 7, France

E-mail: cyril.houdayer@ens-lyon.fr

S. Popa, UCLA Mathematics Department, Box 951555, Los Angeles, CA 90095-1555, U.S.A.

E-mail: popa@math.ucla.edu

S. Vaes, Department of Mathematics, KU Leuven, Celestijnenlaan 200B, 3001 Leuven, Belgium

E-mail: stefaan.vaes@wis.kuleuven.be 